\title{
Utilidad de la biopsia por congelación para el diagnóstico y tratamiento de la mucormicosis. Reporte de un caso y revisión de la literatura
}

\author{
Samuel David Morales-Naranjo'; Juan Eduardo Rocha-Aguirre²; Rocío Paola Prieto- \\ Pérez ${ }^{3}$
}

\section{RESUMEN}

Introducción: La mucormicosis hace referencia a las infecciones por hongos de la familia Mucoraceae, del orden Mucorales. Existen tres géneros importantes responsables de las infecciones humanas: Rhizopus, Mucor y Absidia. Se ha descrito mucormicosis rinocerebral, sinonasal, pulmonar, gastrointestinal, cutánea y diseminada. Generalmente afecta a pacientes inmunodeprimidos o con algún grado de compromiso del sistema inmunológico. Materiales y métodos: reporte de caso de una paciente femenina de 24 años, con cuadro clínico de aproximadamente 1 mes de evolución de otalgia izquierda, que recibió diferentes manejos antibióticos sin mejoría del cuadro. Refiere que se le realizó un procedimiento en cara y arcada superior dental izquierda con posterior cambio de la coloración de la piel, palidez y necrosis. Se realiza diagnóstico de sepsis de tejidos blandos y diabetes mellitus de novo. Es valorado por el servicio de dermatología, donde toman biopsia para cortes por congelación, en los cuales se reconocen filamentos hialinos en forma de cintas, hifas de paredes irregulares y de diámetro variable, algunas irregularmente ramificadas, en ángulos mayores de $45^{\circ}$ y escasos septos, los cuales resaltan con la coloración de ácido peryódico de Schiff (PAS) en los posteriores cortes en parafina. El objetivo del presente artículo es describir la utilidad de la biopsia por congelación en el diagnóstico precoz de la mucormicosis.

Discusión: el uso de la biopsia por congelación en el contexto de la mucormicosis ha mostrado ser una herramienta valiosa para la evaluación intraoperatoria de la piel y los tejidos blandos, con grandes impactos en la efectividad del tratamiento y la supervivencia, por lo que debe ser utilizada en conjunto con las características clínicas al momento de la toma de decisiones.

PALABRAS CLAVE: Biopsia por congelación; Coloraciones de histoquímica para hongos; Evaluación intraoperatoria; Infecciones fúngicas invasivas; Mucormicosis sinonasal.

1. Médico Patólogo, Hospital de San José/Instituto Nacional De Cancerología, instructor programa de patología Fundación universitaria de ciencias de la Salud (Fucs)/Hospital De San José, Sociedad de Cirugía de Bogotá. ORCID https://orcid.org/oooo-0001-8473-3073

2. Médico Patólogo y Epidemiólogo, Fundación universitaria de ciencias de la Salud (Fucs), Fellow Dermatopatología Universidad CES, Medellín Colombia. ORCID https://orcid.org/oooo-0002-8068-5702

3. Médico especialista en Sistemas de garantía de calidad y auditoria de servicios de salud, Residente de tercer año del programa de patología Fundación universitaria de ciencias de la Salud (Fucs)/Hospital De San José, Sociedad de Cirugía de Bogotá. ORCID https://orcid.org/oooo-00o2-9639-2111

Correspondencia: Juan Eduardo Rocha-Aguirre; email: md.juanrocha@gmail.com

Recibido: 29/11/19; aceptado: 03/07/20

Cómo citar: Morales, SD; Rocha, JE; Prieto, RP. Diagnóstico de Mucormicosis mediante biopsia por congelación. Rev Asoc Colomb Dermatol. Vol 28(4): octubre - diciembre, 2020, 357-363. DOI: https://doi.org/10.29176/2590843X.1555

Financiación: ninguna, conflictos de interés: ninguno 


\section{UTILITY OF FROZEN SECTIONS BIOPSY FOR THE DIAGNOSIS AND TREATMENT OF MUCORMYCOSIS. CASE REPORT AND REVIEW OF THE LITERATURE}

\section{SUMMARY}

Introduction: Mucormycosis refers to a fungal infection by the Mucor family. There are three main types of human infections: Rhizobium, Mucor and Absidia. Mucosal spread has been describe in Immunosuppressed patients or patients with some degree of damage to the immune system.

Materials and methods: Case report of a 24-year-old patient that consulted for 1 month of pain in the left ear, previously she had a dental procedure. Patient received different antibiotics without medical response. She has the diagnosis of sepsis, soft tissue infection and new-onset diabetes. She was evaluate by the dermatology service and we took a biopsy to evaluate frozen tissue. Were identified hyphae with bands, irregular walls and variable diameters, some irregular branches, and transparent filaments with an angle greater than $45^{\circ}$. The main objective of this article is to describe the role of frozen biopsy in the early diagnosis of mucormycosis.

Discussion: The use of biopsy and frozen tissue evaluation in mucormycosis has proven to be a valuable tool for intraoperative assessment of skin and soft tissues. It has a great influence on the effect of the treatment.

KEY WORDS: Frozen section biopsy; Fungal histochemistry stains; Intraoperatory evaluation; Invasive fungal infections; Sinonasal mucormycosis.

\section{CASO CLÍNICO}

Paciente femenina de 24 años, procedente de Puerto Boyacá, en el departamento de Boyacá, Colombia, con cuadro clínico de aproximadamente 1 mes de evolución consistente en otalgia izquierda con múltiples consultas asociadas, por lo que recibió diferentes manejos antibióticos que incluían: amoxicilina, azitromicina, ceftriaxona y amoxicilina + clavulanato sin mejoría del cuadro clínico. Posterior a esto la paciente consultó nuevamente por empeoramiento de los síntomas, en esta ocasión presentando edema facial en hemicara izquierda, dolor ocular, edema palpebral, cefalea y rinorrea, por lo que es hospitalizada con manejo hospitalario con clindamicina y ciprofloxacina. Dentro de los paraclínicos evaluados se reportó un hemograma con leucocitosis, neutrofilia y glucometría con 493 mg/dL.

Al interrogar de nuevo a la paciente comentó que se le realizó un procedimiento en cara y arcada superior dental izquierda, con cambio de la coloración de la piel con palidez y necrosis (figura 1). Ante los hallazgos, la paciente es diagnosticada con cuadro de sepsis de tejidos blandos secundaria a celulitis postseptal con riesgo de meningitis y diabetes mellitus de novo, que debutó con cetoacidosis diabética. Se inicia antibiótico endovenoso con vancomicina, meropenem e insulina cristalina. Es valorado por el servicio de dermatología, donde tomaron biopsias para cortes por congelación.

En los cortes por congelación se reconoció la presencia de filamentos hialinos en forma de cintas, con hifas de paredes irregulares y de diámetro variable, algunas irregularmente ramificadas, en ángulos mayores de $45^{\circ}$ (figura 2), los cuales resaltan con la coloración de ácido peryódico de Schiff (PAS) en los posteriores cortes realizados en parafina (figura 3) y los cuales resaltan de manera débil y focal con la coloración de plata de Gomori en los mismos cortes en parafina (figura 4). Cabe resaltar que para la confirmación del diagnóstico se 


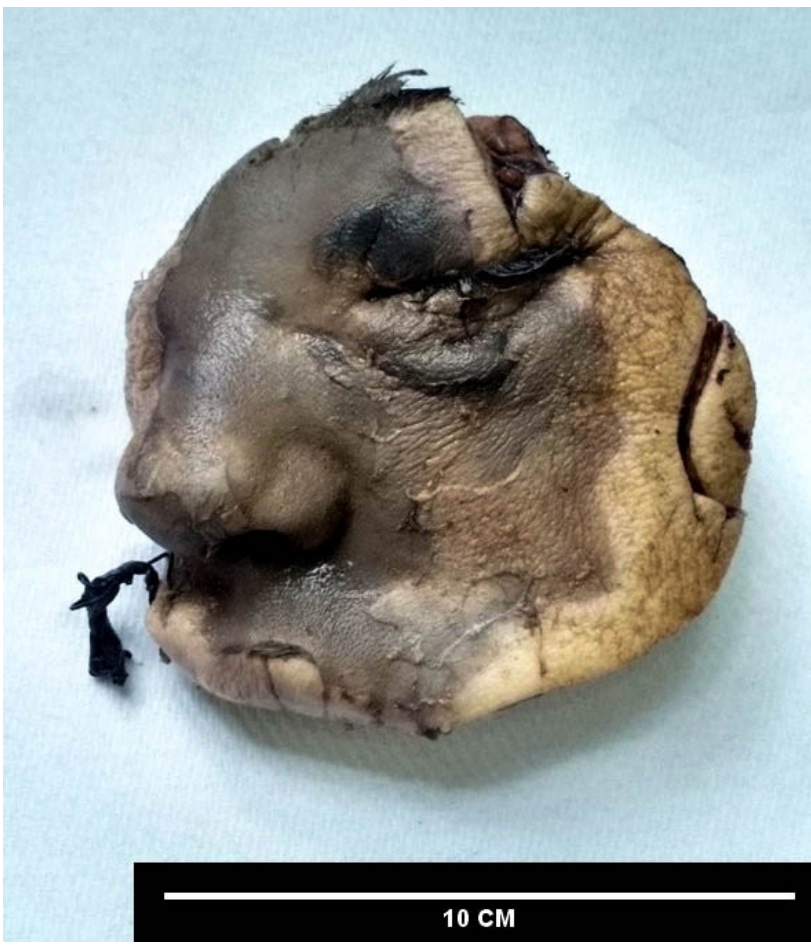

Figura 1. Producto de resección de area necrótica en cara, previo diagnóstico de mucormicosis angioinvasiva mediante cortes por congelación.

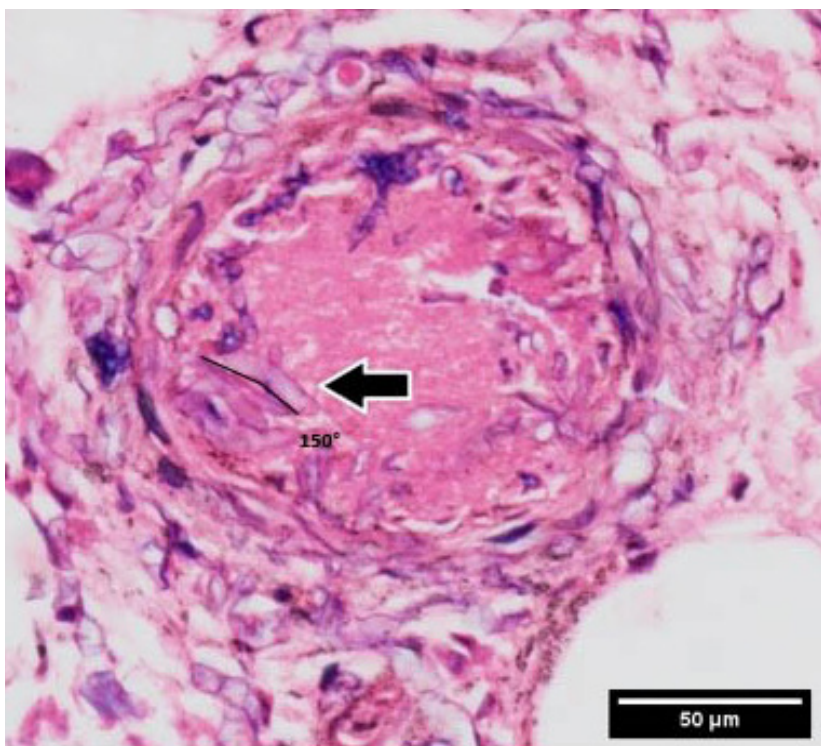

Figura 2. Hifas de paredes irregulares y de diámetro variable, algunas irregularmente ramificadas, en ángulos mayores de $45^{\circ}$, a nivel de un vaso sanguíneo (angioinvasión). Aumento a $40 \mathrm{x}$, con coloración de hematoxilina y eosina (H\&E). 


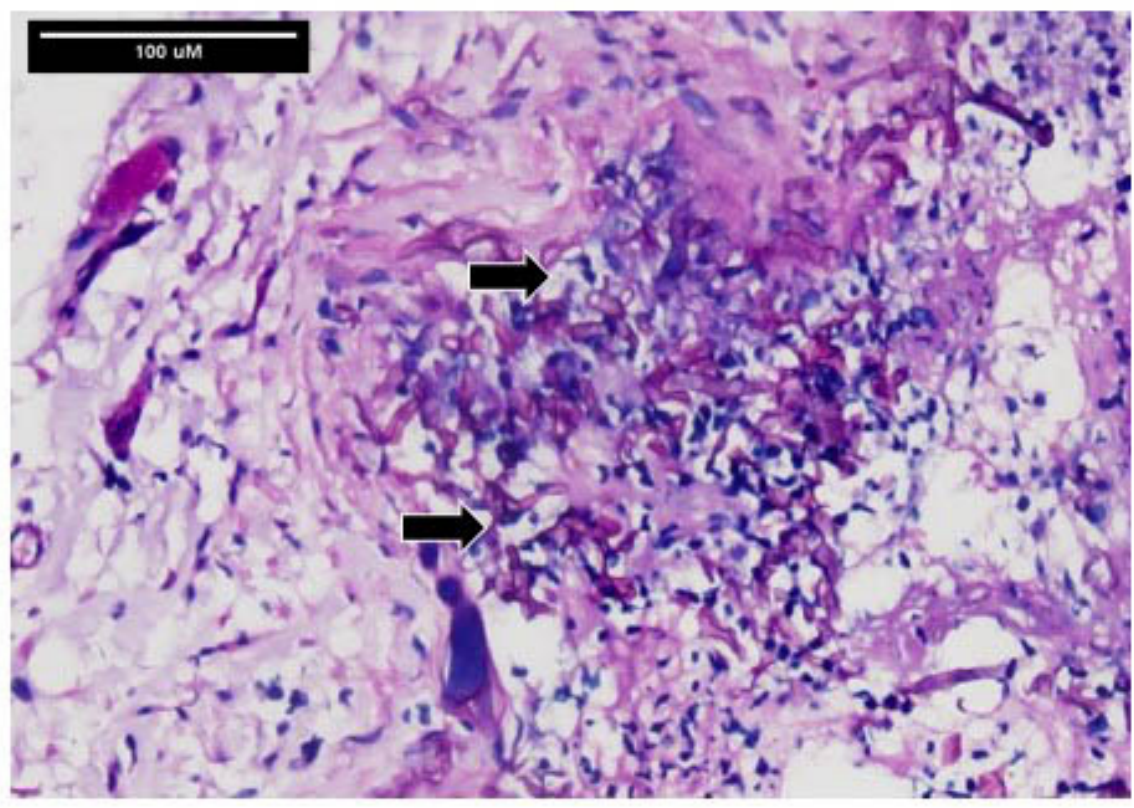

Figura 3. Coloración de ácido peryódico de Schiff (PAS) en cortes en parafina, resalta las hifas (flechas negras) (aumento 40x).

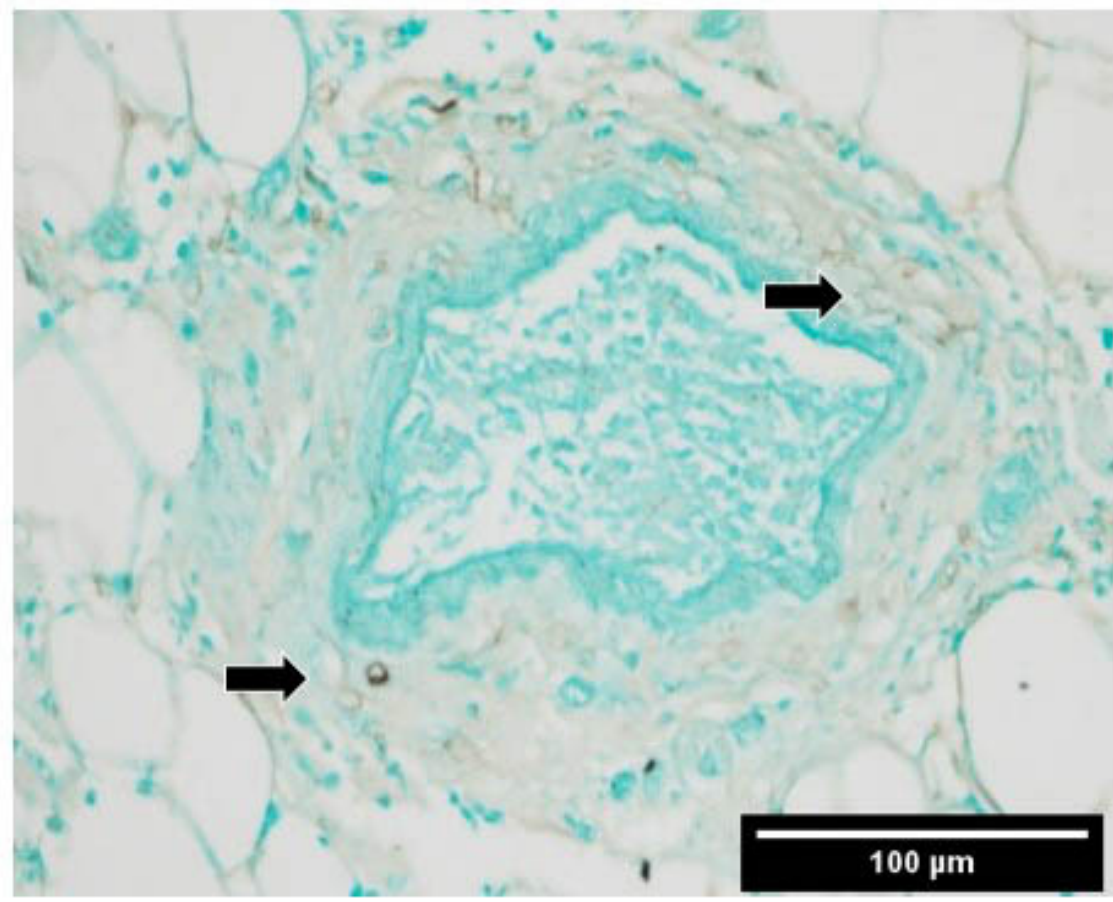

Figura 4. Coloración de Gomori metamina de plata, de uso rutinario para detectar microorganismos en cortes en parafina. En este caso, se observa la tinción de manera débil, casi imperceptible y focal de las hifas (flechas negras), aumento a 40x. 


\section{Puntos clave}

- Dentro de los géneros más comunes encontrados en infecciones en humanos se encuentran: Rhizopus spp., Mucor spp., Rhizomucor spp.

- Factores de riesgo: inmunosupresión, diabetes mellitus, inyecciones, traumatismos, quemaduras, desnutrición, sobrecarga de hierro.

- En el estudio histopatológico se observan hifas gruesas (5-20 $\mu \mathrm{m})$, no pigmentadas de paredes delgadas con algunos septos y ramificaciones en ángulo recto con presencia de angioinvasión y necrosis tisular.

- Las hifas pueden resaltar con coloraciones de plata y acido peryódico de Schiff en las preparaciones de tejidos. Sin embargo, la correlación con la clínica, cultivos y/o estudios moleculares en necesaria.

- El diagnóstico diferencial histopatológico incluye: aspergilosis, candidiasis y la hialohifomicosis.

realizó un cultivo para hongos en los tejidos y la secreción nasal de la paciente, los cuales fueron positivos para crecimiento de Rhizopus spp.

\section{DISCUSIÓN}

La mucormicosis hace referencia a las infecciones no oportunistas por hongos de la familia Mucoraceae, una de las que constituyen el orden Mucorales. Existen tres géneros importantes responsables de las infecciones humanas: Rhizopus, Mucor y Absidia. Se ha descrito mucormicosis rinocerebral, pulmonar, gastrointestinal, cutánea y diseminada ${ }^{(1-4)}$. Generalmente afecta a paciente inmunodeprimidos o con algún grado de compromiso del sistema inmunológico, como los pacientes con patologías hematológicas malignas (leucemias y linfomas), trasplantados, enfermedad renal crónica, pacientes diabéticos, malnutridos, neonatos, politraumatizados o quemados ${ }^{(5-7)}$.

En términos generales, en la mayoría de los casos existe algún factor predisponente evidente a la enfermedad; sin embargo, se han descrito casos de estados de inmunodeficiencia ocultos, sobre todo en el contexto de traumatismos ${ }^{(8,9)}$. En estas situaciones se deben descartar factores de riesgo como la diabetes, las discrasias sanguíneas, los antecedente de consumo de drogas de abuso y las alteraciones inmunológicas (alteraciones del sistema de complemento, distribución de inmunoglobulinas y poblaciones linfocitarias); no obstante, su incidencia se ha incrementado de forma significativa en pacientes $\sin$ inmunodeficiencias ${ }^{(10)}$.

En los últimos 30 años, el pronóstico para los pacientes con mucormicosis rinocerebral (MRC) y sinonasal (MSN) ha mejorado considerablemente, con reporte de tasas de supervivencia hasta del $85 \%{ }^{(7)}$. Sin el adecuado abordaje, que incluye diagnóstico temprano y tratamiento, la enfermedad evoluciona drásticamente con celulitis facial, necrosis de la mucosa nasal y senos paranasales, parálisis facial y pérdida de la visión, lo que conduce rápidamente a la extensión intracraneal y posteriormente a la muerte ${ }^{(11)}$. La terapia exitosa incluye el control rápido del proceso de la enfermedad subyacente, la terapia antimicótica sistémica con anfotericina B liposomal y el desbridamiento quirúrgico agresivo del tejido infectado ${ }^{(12,13)}$. El control del trastorno primario es probablemente el determinante más importante de la supervivencia. En un estudio, la tasa de supervivencia disminuyó al 60\% en pacientes con diabetes mellitus y solo al 20\% en aquellos con otros trastornos sistémicos ${ }^{(14)}$. Hasta hace poco, muchos consideraban que la exenteración orbitaria era la terapia quirúrgica de elección para la MRC. Sin embargo, un diagnóstico muy temprano de MRC y MSN en realidad puede evitar tales mutilaciones con efectividad de la terapia antibiótica con anfotericina B, por lo cual se ha recomendado el uso de la biopsia por congelación ${ }^{(13,15)}$.

El primer reporte de utilización de biopsia por congelación intraoperatoria en el ejercicio de la medicina fue realizado por el patólogo Willian Welch en 1891, en el Hospital Johns Hopkins, para abordar un caso sospechoso de cáncer de mama. Para dicho caso se utilizó un micrótomo de congelación de dióxido de carbono. El caso pertenecía al Dr. William Halsted ${ }^{\left({ }^{16)} \text {. }\right.}$

Existen datos que sugieren el uso de la biopsia por congelación en casos de MRC, en tejidos blandos y MSN en dos escenarios: el primero para realizar un diagnóstico rápido que permita el inicio inmediato de la terapia antifúngica; y el segundo después del control histopatológico para guiar la erradicación completa del tejido 
infectado durante el desbridamiento quirúrgico ${ }^{(15,17)}$. En cuanto a la determinación de márgenes durante el proceso de desbridamiento de tejidos blandos, se ha documentado un buen rendimiento operativo de los cortes por congelación, con valores de sensibilidad entre $68,4 \%$ y $89 \%$ y $100 \%$ de especificidad. Los valores predictivos negativos y positivos se encuentran entre $70,0 \%$ y $100 \%$, respectivamente, con variaciones del rendimiento debido a la inflamación aguda y a la necrosis, que con el uso de coloraciones especiales de histoquímica se ha logrado mejorar la sensibilidad $(100 \%)$ a expensas de la especificidad $(42,9 \%)^{(18)}$. Se ha descrito la utilidad de la coloración con azul de toluidina para la detección de Mucorales, con menor rendimiento operacional para las coloraciones de Gomori y PAS ${ }^{(15)}$. En el presente caso el uso de la coloración de PAS fue de gran utilidad en el estudio histológico en los posteriores cortes en parafina, tanto en los controles de biopsia de congelación como en el producto de la resección final, que ayudó a resaltar las figuras micóticas.

A pesar de la gran utilidad y de las ventajas de la biopsia por congelación en el abordaje precoz de las micosis invasivas, el estudio de especies de hongos de la familia Mucoraceae en cortes de congelación puede considerarse un reto para los patólogos; inclusive patólogos inexpertos podrían pasar por alto el diagnóstico de mucormicosis en este tipo de cortes debido a que los organismos mucorales eventualmente muestran algunas formas poco comunes, además de que los tejidos generalmente son necróticos y hemorrágicos, lo cual dificulta el examen histopatológico y disminuye drásticamente el rendimiento operativo de la prueba, con valores de sensibilidad menores del $75 \%$, lo que aumenta la tasa de falsos negativos ${ }^{(17)}$. En todos los casos se debe correlacionar con los posteriores hallazgos en cortes en parafina, tanto de los controles del tejido sometido a cortes para congelación como en el producto de la resección final, ya que se ha descrito un óptimo rendimiento operativo en este tipo de cortes, que mejora, de este modo, la precisión y exactitud del estudio histopatológico en las micosis invasivas ${ }^{(19)}$. De la mano de esto, es importante enfatizar en que se debe realizar un cuidadoso procesamiento macroscópico del tejido en todos los casos, sobre todo en aquellos que van a ser sometidos al proceso histotécnico para cortes en parafina, debido a que el tratamiento agresivo del tejido puede hacer que los elementos fúngicos frágiles no sean viables y generar falsos negativos ${ }^{(20)}$.

Al evaluar los cortes por congelación o en parafina teñidos tanto con hematoxilina y eosina como con tinciones especiales de PAS y Gomori, la principal diferenciación morfológica entre los géneros Mucorales y otros hongos se debe realizar con aquellos que producen hifas no pigmentadas en el tejido, incluidas Aspergillus spp., otras especies de hongos hialinos septados (como Fusarium y Scedosporium) y Candida spp. ${ }^{(21)}$. Sin embargo, el examen cuidadoso permite un diagnóstico precoz adecuado ${ }^{(6,9,15)}$, con grandes impactos en la supervivencia y efectividad del tratamiento ${ }^{(17}$, ${ }^{18)}$. La presencia de frecuentes tabiques y de ramificaciones en ángulos agudos debe sugerir el diagnóstico de Aspergillus spp. ${ }^{(22)}$. La tinción débil y focal de las hifas con Gomori e inclusive negativa en cortes histológicos en parafina debería sugerir mucormicosis ${ }^{(23,24)}$. Dada esta complejidad, la prueba de referencia para el diagnóstico confirmatorio sigue siendo tanto el cultivo de la muestra de tejido como las pruebas moleculares, bien sea PCR con secuenciación utilizando primers 28S rRNA o secuenciación de nueva generación ${ }^{(11,19,25)}$. Para detectar infecciones duales mediante Mucorales y otros hongos, es recomendable el uso de técnicas de inmunohistoquímica, hibridación in situ o PCR ${ }^{(25)}$.

\section{CONCLUSIONES}

El uso de la biopsia por congelación en el contexto de la mucormicosis para la evaluación intraoperatoria de la piel y los tejidos blandos para el diagnóstico, la determinación de los márgenes quirúrgicos y la instauración temprana del tratamiento antibiótico ha mostrado ser una herramienta valiosa, con grandes impactos en la efectividad del tratamiento y supervivencia. Consideramos que los hallazgos histopatológicos deben ser utilizados en constructo junto con las características clínicas al momento de la toma de decisiones en el abordaje inicial y correlacionados finalmente con el resultado de cultivos y pruebas moleculares confirmatorias.

\section{REFERENCIAS}

1. García CA, García E, Gómez C, Castillo SA. Mucormicosis gastrointestinal en paciente diabético; reporte de un caso y revisión de la literatura. Infectio. 2012;16(3S):108-13.

2. Botero JC, Moreno LF, Caparroso LC. Mucormicosis rinocerebral en paciente con cefalea y diabetes mellitus. Infectio. 2008;12(1):298-303.

3. Tapia O,Chahín C, ConchaC.Mucormicosiscutánea primaria: a propósito de dos casos. Revisión de la 
literatura. Revista Chil Infectol. 2011;28(3):269-73. doi: 10.4067/So716-10182011000300012

4. Vergara DIN, Barragán G, Bonifaz A, Núñez C. Mucormicosis pulmonar en un paciente con trasplante renal y hemoptisis incoercible. Rev Iberoamericana Micol. 2017;34(4):233-6.

5. Pak J, Tucc V, Vincent A, Sandin R, Greene J. Mucormycosis in immunochallenged patients. J Emerg Trauma Shock. 2008;1(2):106-13. doi: 10.4103/0974-2700.42203

6. Sarvestani S, Pishdad G, Bolandparvaz S. Epidemiology and Clinical Characteristics of Mucormycosis in Patients with Leukemia; A 21-year Experience from Southern Iran. Bull Emerg Trauma. 2014;2(1):38-43.

7. Noorifard M, Sekhavati E, Khoo H, Hazraty I, Tabrizi R. Epidemiology and clinical manifestation of fungal infection related to Mucormycosis in hematologic malignancies. J Med Life. 2015;8(Spec Iss 2):32-37.

8. Skiada A, Groll A, Zimmerli S, Dupont B, Lagrou K, Lass-Florl C, et al. Zygomycosis inEurope: analysis of 230 cases accrued by the registry of the European Confederation of Medical Mycology (ECMM) Working Group on Zygomycosis between2005 and 2007. Clin Microbiol Infect. 2011;17(12):1859-67. doi: 10.1111/j.1469-0691.2010.03456.x

9. Deja M, Weber-Carstens S, Lehmann TN, Adler A, Ruhnke M, Tintelnot K. Gastrointestinal zygomycosis caused by Mucor indicus in a patient with acute traumaticbrain injury. Med Mycol. 2006;44(7):683-7.doi:10.1080/13693780600803888

10. Pozo JC, Ponte A, Pozo C, Robles JC, Linares MJ. Mucormicosis diseminadas en pacientes sin inmunodeficiencias: una enfermedad que también existe. Rev Iberoamericana Micol. 2015;32(2):6370. doi: 10.1016/j.riam.2014.01.006

11. Kradin RL. Diagnostic pathology of infectious disease. Filadelfia: Saunders; 2018.

12. Zahoor BA, Piercey J, Wall D, Tetsworth K. A surgical approach in the management of mucormycosis in a trauma patient. Annals R Coll Surg Engl. 2016;98(8):e173-7. doi: 10.1308/rcsann.2016.0221

13. Singh V, BansalC, Kaintura M. Sinonasal $\mathrm{Mu}-$ cormycosis: A to Z. Indian J Otolaryngol Head Neck Surg. 2019;71(Suppl 3):1962-71. doi: 10.1007/ s12070-018-1384-6

14. 14. Espellberg B, Walsh TJ, Kontoyiannis DP, Edwards Jr J, Ibrahim AS. Recent advances in the management of mucormycosis: from bench to bedside. Clin Infect Dis. 2009;48(12):1743-51. doi: 10.1086/599105
15. Hofman V, Castillo L, Bétis F, Guevara N, GariToussaint M, Hofman P. Usefulness of frozen section in rhinocerebral mucormycosis diagnosis and management. Pathology. 2003;35(3):212-6. doi: 10.1080/0031302031000123173

16. Delgado JA. Realidad en biopsias por congelación. Universitas Médica. 2007;48(1):37-45.

17. Hennessy M, McGinn J, White B, Payne S, Warrick J, Crist H. Frozen section as a rapid and accurate method for diagnosing acute invasive fungal rhinosinusitis. Otolaryngol Head Neck Surg. 2018;159(3):576-80. doi: 10.1177/0194599818770615

18. Zimmermann N, Hagen M, Schrager J, HebbelerClarck R, Masineni S. Utility of frozen section analysis for fungal organisms in soft tissue wound debridement margin determination. Diagn Pathol. 2015;10(1):188. doi: 10.1186/s13000-015-0423-9

19. Milner DA. Diagnostic Pathology: Infectious Diseases. 2.a edición. Filadelfia: Elsevier Health Sciences; 2019.

20. Son HJ, Song JS, Choi S, Jung J, Jae M, Chong Y, et al. A comparison of histomorphologic diagnosis with culture- and immunohistochemistry-based diagnosis of invasive aspergillosis and mucormycosis. Infect Dis (Lond). 2020;52(4):279-83. doi: 10.1080/23744235.2020.1716063

21. Ribes JA, Vanover-Sams CL, Baker DJ. Zygomycetes in human disease. 2000;13(2):236-301. doi: 10.1128/ cmr.13.2.236-301.2000

22. Guarner J, Brandt M. Histopathologic diagnosis of fungal infections in the 21st century. 2011;24(2):247-80. doi: 10.1128/CMR.00053-10

23. Hofman V, Dhouibi A, Butori C, Padovani B, Gari-Toussaint M, Garcia D, et al. Usefulness of molecular biology performed with formaldehyde-fixed paraffin embedded tissue for the diagnosis of combined pulmonary invasive mucormycosis and aspergillosis in an immunocompromised patient. Diagn Pathol. 2010;5(1):1. doi: 10.1186/1746-1596-5-1

24. Sharifipour F, Rezaeetalab F, Naghibi M. Pulmonary fungal infections in kidney transplant recipients: an 8-year study. Transplant Proc. 2009;41(5):1654-6. doi: 10.1016/j.transproceed.2009.02.072

25. Larkin PM, Lawson K, Contreras D, Le C, Trejo $\mathrm{M}$, Realegeno S, et al. Amplicon-Based NextGeneration Sequencing for Detection of Fungi in Formalin-Fixed, Paraffin-Embedded Tissues: Correlation with Histopathology and Clinical Applications. J Mol Diagn. 2020;22(10):1287-93. doi: 10.1016/j.jmoldx.2020.06.017 